\section{Role of Sertraline as a Mono-therapy in Treatment of Irritable Bowel Syndrome and Associated Psychological Problems: A Single- blinded Randomized Controlled Trial}

\section{Abstract}

Background: Irritable bowel syndrome (IBS) is considered one of the most common functional gastrointestinal disorders. The pathogenesis of IBS is still unknown, as it has both physiological and psychological factors. Being a multifactorial disorder, the treatment of IBS is thereby challenging and also conflicting. Conventional therapy might have an effect in mild and recently diagnosed cases but for some while and some extent. So, antidepressant medications were proposed to treat IBS with long lasting effect. This study aimed to compare effect of sertraline to placebo in treatment of IBS and its comorbid anxiety and/or depressive symptoms.

Methods: 55 patients were recruited from outpatient clinics at Assiut University Hospitals and were diagnosed as irritable bowel syndrome according to Rome III criteria. Patients underwent Hamilton rating scale for anxiety and depression to assess their level of anxiety and depression respectively. Patients were divided into two groups, one group of patients received sertraline and the second group received placebo. Blinding was concealed throughout the clinical trial. Assessment of patients was done after four weeks regarding their bowel symptoms, anxiety, and depressive symptoms.

Results: Sertraline group showed significant reduction of bowel symptoms in 20 patients $(66.67 \%)$ versus 8 patients $(32 \%)$ who received placebo $(P=0.001)$. Also, there was significant reduction in anxiety and depressive scores in Hamilton rating scale for anxiety and depression respectively $(P=0.001)$.

Conclusion: Sertraline is superior to placebo in treatment of irritable bowel syndrome especially in those who have comorbid anxiety and depression.

Keywords: Irritable bowel syndrome; Sertraline; Psychosomatic

\section{Ihab M Saleh ${ }^{1 *}$, Khaled O Mohamed ${ }^{1}$, Muhammad A El-Masry² and Nageh F Kamel ${ }^{1}$}

1 Department of Neuro-psychiatry, Assiut University, Assiut, Egypt

2 Department of Internal Medicine, Division of Gastroenterology, Assiut University, Assiut, Egypt

\section{*Corresponding author: Ihab M Saleh \\ ”ihab.mohamed@med.aun.edu.eg}

Department of Neuro-psychiatry, Faculty of Medicine, Assiut University, Assiut, Egypt.

Tel: $+20-88-2357007$

Fax: $+20-2-37484536$

Citation: Saleh IM, Mohamed KO, El-Masry M, Kamel NF (2017) Role of Sertraline as a Mono-therapy in Treatment of Irritable Bowel Syndrome and Associated Psychological Problems: A Single-blinded Randomized Controlled Trial. J Neurol Neurosci Vol.8 No. 5:218

Received: August 23, 2017; Accepted: September 18, 2017; Published: September 22, 2017

\section{Introduction}

One of the commonest functional gastro-intestinal disorders is irritable bowel syndrome (IBS), as its worldwide prevalence is about $5-20 \%$, depending on Rome III criteria [1]. It is more common in younger individuals especially in women [2]. Patients who have IBS are more liable to have sickness-related absences than those who do not have bowel symptoms [3]. A recent burden-of-IBS in the United States estimated, and found that its direct cost is almost 1 billion Dollars and indirect cost is another 50 million Dollars [4]. Moreover, health-care resources consumption of patients with IBS is about $>50 \%$ [5]. Therefore, treatment of IBS effectively is crucial whether for the individuals or for health-care systems and the whole society.

The exact cause of IBS remains unknown, which means there is no single unifying explanation for the bowel symptoms toward which therapy can be targeted. Patients with IBS show abnormal central processing of pain [6], visceral hypersensitivity [7,8], and higher levels of psychological comorbidity when compared with healthy controls without bowel symptoms $[9,10]$. As a result, antidepressants have been introduced as treatments for IBS as 
they have pain-modifying properties $[11,12]$. Serotonin $(5-H T)$, through the 5-HT3 and 5-HT4 receptors, plays a vital role in the control of gastrointestinal motility, secretion and sensation. Furthermore, recent observations found that the plasma 5-HT concentrations are reduced in IBS patients [13].

Several studies demonstrated that IBS patients have abnormal personality as well as higher scores of anxiety and depression [14-16]. However, many studies evaluated antidepressants effectiveness on IBS; their clinical evidence is still controversial. The most commonly used antidepressants are selective serotonin reuptake inhibitors (SSRI) such as fluoxetine and citalopram and tricyclic antidepressants (TCA) such as imipramine and amitriptyline.

As a selective serotonin reuptake inhibitor, sertraline acts by blocking the reuptake of serotonin in CNS synaptic clefts, thus increasing serotonin levels in the brain which is associated with its psychiatric effects. Sertraline was approved for use in the United States in 1991, and it remains in wide use, with almost 40 million prescriptions being filled yearly. It is well known for treating depression and anxiety disorders. Also, it considered one of selective serotonin reuptake inhibitors that has a pain regulatory mechanism, so it is commonly used in psychosomatic disorders as well [17].

This study compared the effectiveness of sertraline to placebo effect (conventional therapy as anti-spasmodic and antidiarrhoeal medications) regarding their effect on bowel symptoms of irritable bowel syndrome as well as anxiety and depressive symptoms associated with.

\section{Methods}

The present study was a prospective sertraline, placebo randomized clinical trial of 1 month 's duration with single blinded treatment and blinded outcome measures. It consisted of 55 patients who recently diagnosed as irritable bowel syndrome (IBS) according to Rome III criteria who were recruited within 4 weeks from outpatient clinics at Assiut University Hospitals.

\section{Sample size}

In case control studies on IBS, a $50 \%$ response to placebo is frequently observed. Furthermore, antidepressants response rates of up to $89 \%$ have been observed in open label studies. Using these numbers and with a power of $80 \%$, a sample size of was obtained for each group.

\section{Subjects and study design}

They were at age group between 18-40 years old. We included only patients who were recently diagnosed as IBS and the duration of illness was not more than 5 years. Moreover, all patients were not treated previously with other selective serotonin reuptake inhibitors. All routine investigations were done for all patients including complete blood count, renal and liver function as well as measurement of the electrolytes. The Hamilton rating scale for anxiety (HAM-A) and Hamilton rating scale for depression (HAM-D) were done for all patients at baseline before beginning of treatment. Patients were divided into two groups, first group which was 30 patients who received sertraline $50 \mathrm{mg}$ and the second group which was 25 patients who received placebo. Blinding was concealed throughout the randomized clinical trial. Patients were followed up for one month after treatment. Reassessment of the patients was done regarding their bowel manifestations. Improvement of bowel manifestation was considered when there was adequate relief of symptoms reported from patients after 4 weeks of a medication. Also, the Hamilton rating scale for anxiety (HAM-A) and Hamilton rating scale for depression (HAM-D) were done for all patients one month after treatment.

\section{Exclusion criteria}

1. Patients who had bowel symptoms but not fulfilling Rome III criteria for diagnosis of irritable bowel syndrome.

2. Patients who were having irritable bowel syndrome for more than 5 years duration.

3. Patients who have severe anxiety and/or depression according to HAM-A and HAM-D respectively.

4. Patients who did not meet the age group.

5. Patients who were receiving any other medications.

6. Patients who had any chronic medical conditions.

\section{Data analysis}

The SPSS version 16 package was used in this study. Descriptive statistics were used to compare the effect of sertraline and placebo on bowel manifestations. Paired samples T-test was used to compare the effect of sertraline and placebo on Hamilton rating scale for anxiety (HAM-A) and Hamilton rating scale for depression (HAM-D) respectively.

\section{Results}

This study consisted of 55 patients of irritable bowel syndrome who were diagnosed according to Rome III criteria. Regarding the demographic data of the patients, the mean age of the patients was $26 \pm 2.1$ years old, and female/male ratio was $1.7 / 1$ as there were 32 female patients versus 18 male patients.

The most common GIT manifestation was abdominal discomfort which found in all patients (100\%), followed by abdominal bloating and distension which found in 36 patients (65.45\%). The third common manifestation was improving of the symptoms with defecation which found in 32 patients (58.18\%), and lastly the least common symptoms was alternating bowel habits which found in 29 patients (52.7\%). Patients with IBS-C were more liable to experience abdominal bloating and improving of the symptoms with defecation, while patients with IBS-D were more liable to experience alternating bowel habits (Figure 1).

The patients were divided into two groups. 30 patients received sertraline $50 \mathrm{mg}$ and 25 patients received placebo. 20 patients 


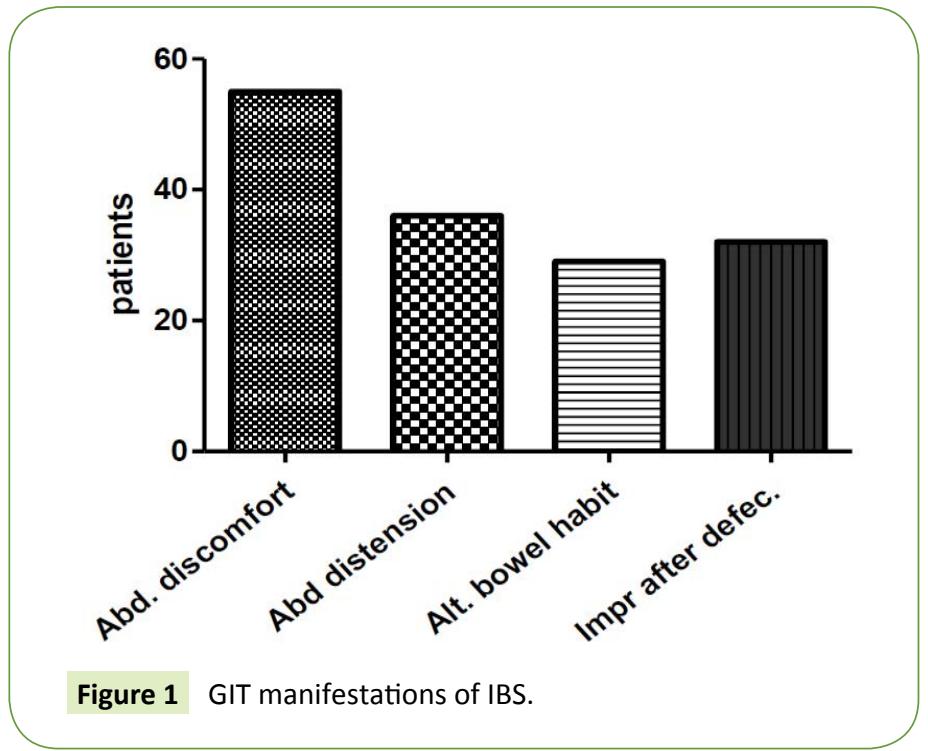

who received sertraline $50 \mathrm{mg}$ showed improvement of their bowel symptoms ( 7 patients had irritable bowel syndrome with predominant diarrhoea and 13 patients had irritable bowel syndrome with predominant constipation) while 10 patients (4 patients had irritable bowel syndrome with predominant diarrhoea and 6 patients had irritable bowel syndrome with predominant constipation) showed no satisfied improvement of their bowel symptoms.

On the other hand, 8 patients who received placebo showed improvement of their bowel symptoms ( 5 patients had irritable bowel syndrome with predominant diarrhoea and 3 patients had irritable bowel syndrome with predominant constipation) while 17 patients (12 patients had irritable bowel syndrome with predominant diarrhoea and 5 patients had irritable bowel syndrome with predominant constipation) showed no improvement of their bowel symptoms (Table 1 and Figure 2).

The two study groups were compared regarding Hamilton rating scale for anxiety and depression respectively. There was significant reduction in Hamilton rating scale for anxiety (HAM-A) and Hamilton rating scale for depression (HAM-D) after one month in the patient group who were treated by sertraline $50 \mathrm{mg}$. As mean HAM-A was $18.8 \pm 3.9$ before treatment and reached $11.67 \pm 1.9$ after one-month treatment with sertraline. Furthermore, mean HAM-D was $15.67 \pm 3.9$ before treatment and reached $11.6 \pm 4.2$ after one-month treatment with sertraline $(P=0.001)$. Whereas, there was no significant reduction in Hamilton rating scale for anxiety (HAM-A) and Hamilton rating scale for depression (HAM-D) after one month in the patient group who were treated by placebo. As mean HAM-A was $18.5 \pm 2.6$ before treatment and reached $18.2 \pm 2.4$ after one-month treatment with placebo $(P=0.12)$ (Table 2). Moreover, mean HAM-D was $13.4 \pm 2.1$ before treatment and reached $13 \pm 1.7$ after one-month treatment with placebo ( $P=0.15$ ) (Figures 3 and 4 ).
Table 1 Numbers of patients showed improvement of bowel symptoms after one month of the medication.

\begin{tabular}{|c|c|c|c|c|c|c|}
\hline & \multicolumn{3}{|c|}{ Improvement } & \multicolumn{3}{c|}{ No improvement } \\
of bowel symptoms & of bowel symptoms \\
\hline & IBS-D & IBS-C & $\%$ & IBS-D & IBS-C & $\%$ \\
\hline Sertraline (n=30) & 7 & 13 & 66.67 & 4 & 6 & 33.33 \\
\hline Placebo (n=25) & 5 & 3 & 32 & 12 & 5 & 68 \\
\hline
\end{tabular}

Note: IBS-D: irritable bowel syndrome with predominant diarrhea, IBS-C: irritable bowel syndrome with predominant constipation

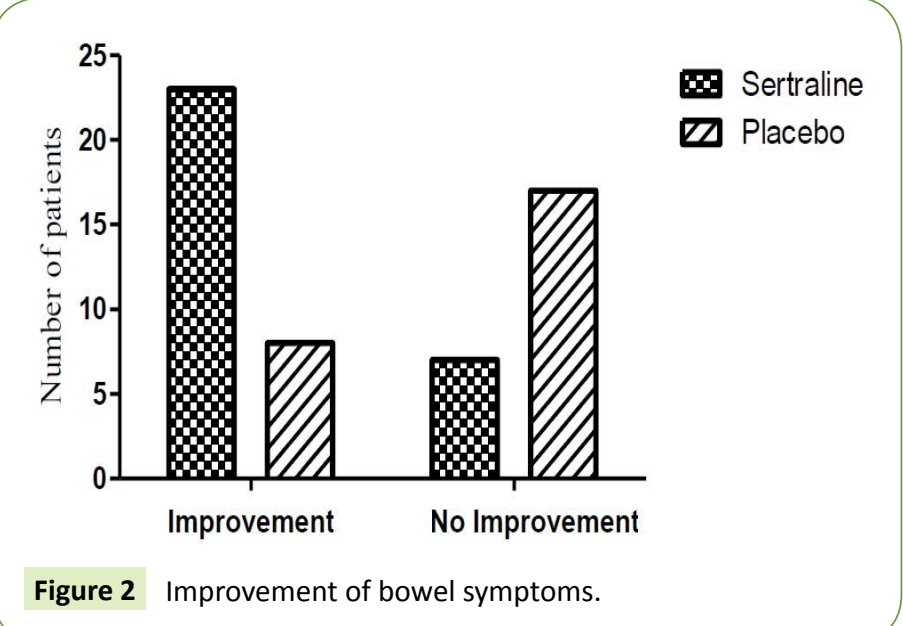

Table 2 The effect of sertraline Vs placebo on HAM-A and HAM-D.
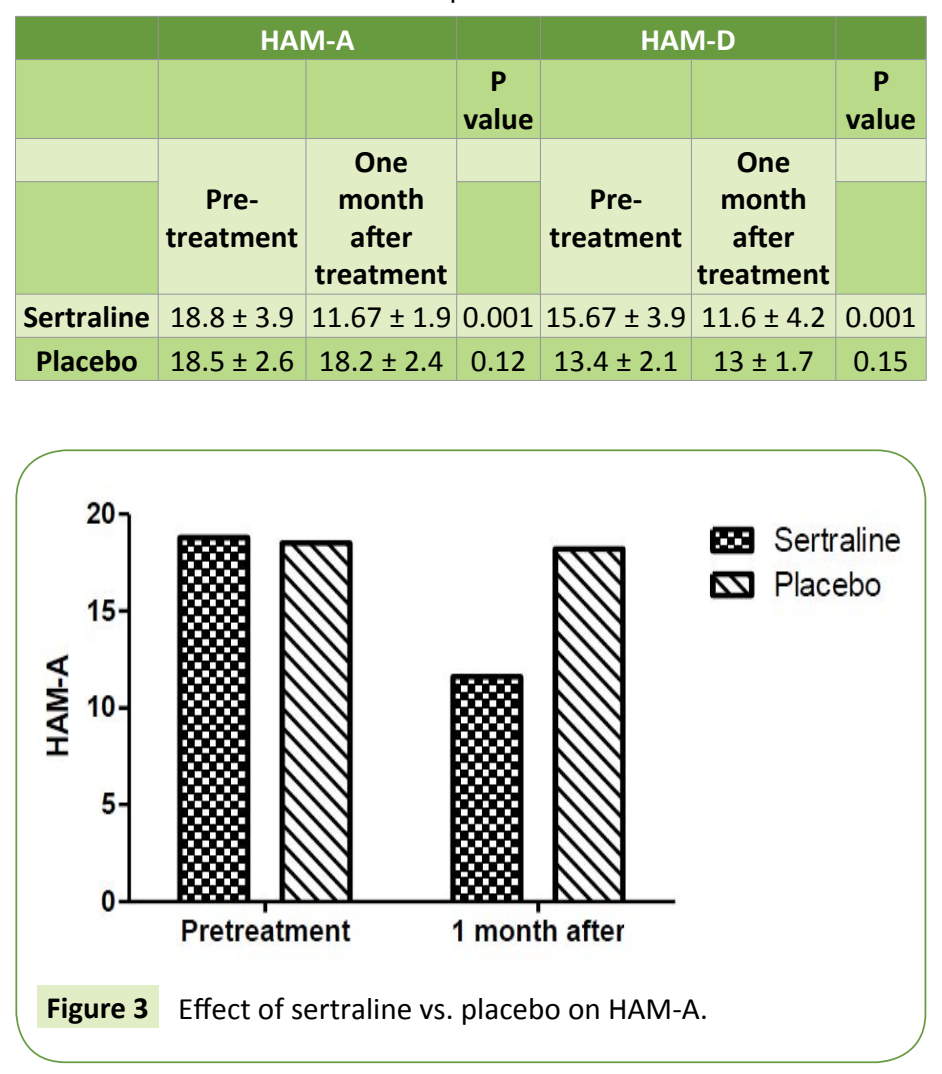


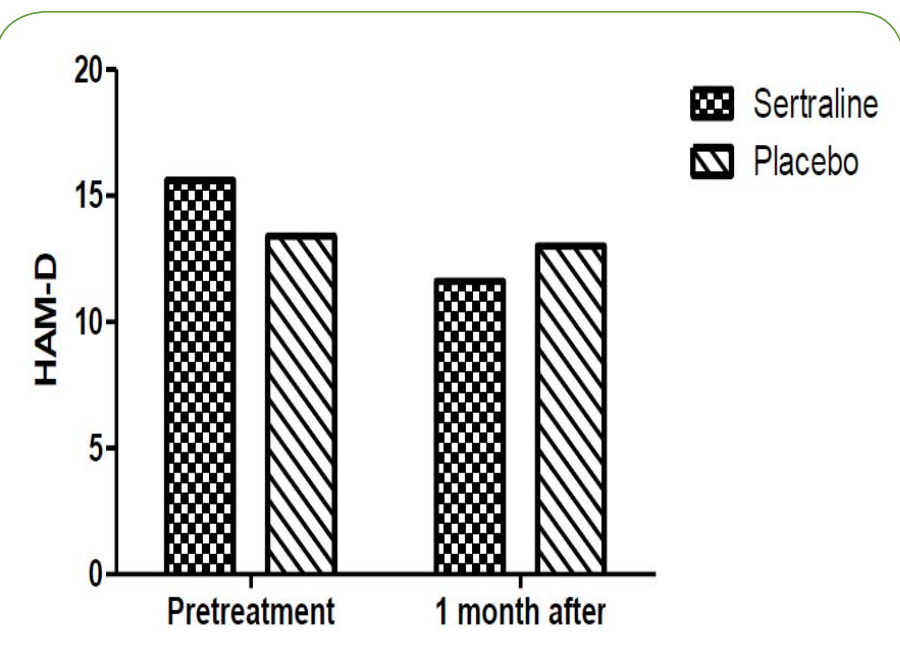

Figure 4 Effect of sertraline vs. placebo on HAM-D.

\section{Discussion}

In literature, six studies discussed the effect of selective serotonin reuptake inhibitors (SSRI) in treatment of IBS with conflicting results. Four studies of paroxetine [18], fluoxetine [19] and citalopram $[20,21]$ reported a modest benefit, while two studies of fluoxetine [22] and citalopram [23] did not.

A meta-analysis of these studies found a relative risk of unimproved symptoms of irritable bowel syndrome (IBS) is $0.62(95 \% \mathrm{Cl}, 0.45$ to 0.87 ) for SSRIs vs. placebo, with significant heterogeneity between these studies [24]. The meta-analysis analyzed data of 113 subjects treated by SSRI and 117 controls.

Differences of results among these six trials may be attributed to the differences of study design as well as population of the patients. Kuiken et al. recruited 40 IBS patients of all subtypes from a tertiary center for a 6-week study of fluoxetine or placebo, patients with depression were excluded in this study [22]. Tabas et al. randomized 81 patients with IBS of all subtypes for a 12week study of a high fiber diet with paroxetine or placebo, patients with depression were not excluded in this study [18]. Vahedi et al. randomized 44 patients with IBS with predominant constipation to fluoxetine or placebo for a 12-week study, but only patients with severe depression were excluded [19]. Tack et al. enrolled 23 IBS patients from a tertiary care center for a crossover trial comparing citalopram vs. placebo, patients with depression were excluded in this study [20].

Ladabaum et al. randomized 27 patients with IBS who were given citalopram $20 \mathrm{mg} /$ day for 4 weeks then citalopram $40 \mathrm{mg} /$ day for another 4 weeks, but psychiatric symptoms were not assessed for these patients [21].

Talley et al. randomized 51 patients with IBS of all types to citalopram (17 subjects), imipramine, or placebo (16 subjects) for 12 weeks, they excluded patients with depression from this trial as well [23].

Features of the trials in which some benefit was detected include the lack of exclusion for depression [18], inclusion of only constipation-predominant IBS patients [19], and a crossover design with patients from tertiary care [20].

The specific drug, dose and duration used in each trial could be important factors, but no clear pattern emerges. The study by Tack et al. is the only one that showed benefit for citalopram [20], while the study by Talley et al. reported identical response rates for citalopram and placebo [23], and Ladabaum et al. found evidence against any substantial benefit of citalopram in treatment of bowel symptoms of IBS [21]. The trials used different definitions for clinical response, and this may explain much of the conflict in the conclusions.

Some of them used adequate relief of IBS symptoms as the primary outcome as Talley et al. In contrast, the other trials assessed global symptom response, overall well-being, or the effect on specific symptoms.

Our study is the first trial which discussed the effect of sertraline in treatment of IBS. We found that sertraline has a significant effect on the bowel symptoms of irritable bowel syndrome (IBS) compared to placebo. The improvement of sertraline was about $66.67 \%$ comparing to placebo which was $32 \%$. Our study demonstrated that the effect of sertraline on IBS with predominant constipation is better than its effect IBS with predominant diarrhoea, this could be explained by increase the intestinal transit with serotonin which may increase diarrhoeal symptoms of IBS [25]. Moreover, this study demonstrated a significant reduction of anxiety and depressive symptoms in sertraline group compared to placebo group which estimated by Hamilton Rating scale for anxiety (HAM-A) and Hamilton Rating scale for depression (HAM-D) respectively.

Sertraline could be effective in treatment of IBS because of the high incidence of co-morbidity of IBS and psychiatric disorders, as its origin might involve physiological as well as psychological processes. Stress is usually associated with onset and severity of symptoms in IBS patients, as the GI tract is a sensitive target to stress. Furthermore, treatment outcome, hospital visits as well as sickness experience are adversely affected by stress, anxiety and depressive disorders as well as maladaptive coping mechanisms [26].

Sensory disorders especially visceral hypersensitivity (VHS) -which is a clinical marker of IBS- has also been considered the patho-physiology of IBS [27]. VHS is represented by the increased sensation of pain when the pelvic colon is distended with an inflated rectal balloon [27]. Hazlett-Stevens et al. who found that anxiety specific to visceral sensations was a strong predictor of IBS [28]. Individuals with anxiety were tend to catastrophize their worries consequences and on the other hand, the hypochondriacal tendency of people with anxiety [28]. These physical symptoms of IBS may reinforce their anxiety and inability to tolerate and cope with these discomforts, thereby forming a vicious circle between these physical and psychological symptoms [29].

So, sertraline effect may be attributed to its antidepressant and anxiolytic effects. Furthermore, sertraline alters the spinal 
modulation of nociceptive stimuli, as well as affects the amygdala activity and the Brain-Gut Axis process by the inhibitory descending pain modulation systems [27].

\section{Conclusion}

Sertraline is effective in treatment of irritable bowel syndrome especially on those who have comorbid depression and/or anxiety. It is recommended to be used in recently diagnosed

\section{References}

1 Lovell RM, Ford AC (2012) Global prevalence of and risk factors for irritable bowel syndrome: a meta-analysis. Clin Gastroenterol Hepatol 10: 712-721.

2 Lovell RM, Ford AC (2012) Prevalence of gastro-esophageal refluxtype symptoms in individuals with irritable bowel syndrome in the community: A meta-analysis. Am J Gastroenterol 107: 1793.

3 Drossman DA (1993) US householder survey of functional gastrointestinal disorders. Dig Dis Sci 38: 1569-1580.

4 Everhart JE, Ruhl CE (2009) Burden of digestive diseases in the United States part I: overall and upper gastrointestinal diseases. Gastroenterology 136: 376-386.

5 Inadomi J, Fennerty MB, Bjorkman D (2003) The economic impact of irritable bowel syndrome. Aliment Pharmacol Ther 18: 671-682.

6 Tillisch K, Mayer EA, Labus JS (2011) Quantitative meta-analysis identifies brain regions activated during rectal distension in irritable bowel syndrome. Gastroenterology 140: 91-100.

7 Moriarty KJ, Dawson AM (1982) Functional abdominal pain: further evidence that whole gut is affected. Br Med J (Clin Res Ed) 284: 16701672.

8 Trimble K (1995) Heightened visceral sensation in functional gastrointestinal disease is not site-specific. Dig Dis Sci 40: 1607-1613.

9 Osterberg E (2000) A population study on irritable bowel syndrome and mental health. Scand J Gastroenterol 35: 264-268.

10 Whitehead WE, Palsson O, Jones KR (2002) Systematic review of the comorbidity of irritable bowel syndrome with other disorders: what are the causes and implications? Gastroenterology 122: 140-1156.

11 McQuay H (1996) A systematic review of antidepressants in neuropathic pain. Pain 68: 217-227.

12 Saarto T, Wiffen PJ (2007) Antidepressants for neuropathic pain. Cochrane Database Syst Rev CD005454.

13 Mayer EA, Bradesi S (2003) Alosetron and irritable bowel syndrome. Expert Opin Pharmacother 4: 2089-2098.

14 Deechakawan W (2014) Anxiety, depression, and catecholamine levels after self-management intervention in irritable bowel syndrome. Gastroenterol Nurs 37: 24-32.

15 Pinto C (2000) Stressful life-events, anxiety, depression and coping in patients of irritable bowel syndrome. J Assoc Physicians India 48: 589-93.

16 lii GRL (2004) Psychosocial factors are linked to functional patients and those with predominant constipation IBS. Further trials needed to discuss the role of sertraline in IBS with exclusion of depression and anxiety. Also, longer duration and larger sample size are needed to be considered in further trials to detect complete relief of symptoms and symptoms relapse if happened.

\section{Conflict of Interest}

No conflict of interest could be declared.

gastrointestinal disorders: A population based nested case-control study. Am J Gastroenterol 99: 350.

17 McLeod AL (2015) Effects of anti-anxiety drugs and other anthropogenic contaminants on fathead minnow behavior and brain chemistry. Thesis submitted to the Graduate School of Clemson University, USA.

18 Tabas G (2004) Paroxetine to treat irritable bowel syndrome not responding to high-fiber diet: a double-blind, placebo-controlled trial. Am J Gastroenterol 99: 914.

19 Vahedi $\mathrm{H}$ (2005) The effect of fluoxetine in patients with pain and constipation-predominant irritable bowel syndrome: A double-blind randomized-controlled study. Aliment Pharmacol Ther 22: 381-385.

20 Tack J (2006) A controlled crossover study of the selective serotonin reuptake inhibitor citalopram in irritable bowel syndrome. Gut 55: 095-1103.

21 Ladabaum U (2010) Citalopram is not-effective therapy for nondepressed patients with irritable bowel syndrome. Clin Gastroenterol Hepatol 81: 42.

22 Kuiken SD, Tytgat GN, Boeckxstaens GE (2003) The selective serotonin reuptake inhibitor fluoxetine does not change rectal sensitivity and symptoms in patients with irritable bowel syndrome: a double blind randomized, placebo-controlled study. Clin Gastroenterol Hepatol 1: 219-228.

23 Talley NJ (2008) Antidepressant therapy (imipramine and citalopram) for irritable bowel syndrome: a double-blind, randomized, placebocontrolled trial. Dig Dis Sci 53: 108-115.

24 Ford AC (2009) Efficacy of antidepressants and psychological therapies in irritable bowel syndrome: systematic review and metaanalysis. Gut 58: 367-378.

25 Longstreth GF (2006) Functional bowel disorders. Gastroenterology 130: 1480-1491.

26 Drossman DA (2000) What determines severity among patients with painful functional bowel disorders \& quest. The Am J Gastroenterol 95: 974-980.

27 Wilder-Smith C (2004) Brain functional magnetic resonance imaging of rectal pain and activation of endogenous inhibitory mechanisms in irritable bowel syndrome patient subgroups and healthy controls. Gut 53: 1595-1601.

28 Hazlett-Stevens H (2003) Prevalence of irritable bowel syndrome among university students: the roles of worry, neuroticism, anxiety sensitivity and visceral anxiety. J Psychosom Res 55: 501-505.

29 Keefer L (2005) Towards a better understanding of anxiety in irritable bowel syndrome: a preliminary look at worry and intolerance of uncertainty. J Cogn Psychother 19: 163-172. 